\title{
Interdigital Piezopolymer Transducers for Time of Flight Measurements with Ultrasonic Lamb Waves on Carbon-Epoxy Composites under Pure Bending Stress
}

\author{
Andrea Bulletti and Lorenzo Capineri \\ Dipartimento Ingegneria dell'Informazione, Università degli Studi di Firenze, Via S. Marta 3, 50139 Firenze, Italy \\ Correspondence should be addressed to Lorenzo Capineri; lorenzo.capineri@unifi.it
}

Received 10 May 2015; Revised 23 July 2015; Accepted 9 August 2015

Academic Editor: Chengkuo Lee

Copyright (C) 2015 A. Bulletti and L. Capineri. This is an open access article distributed under the Creative Commons Attribution License, which permits unrestricted use, distribution, and reproduction in any medium, provided the original work is properly cited.

\begin{abstract}
Interdigital transducers fabricated with piezopolymer film have been realized to excite ultrasonic Lamb waves in a composite laminate subjected to pure bending stresses. Lamb waves were generated and detected in a cross-ply $\left[0^{\circ} / 90^{\circ}\right] 4 \mathrm{~mm}$ thick carbonfiber composite, by using two interdigital transducers in pitch-catch configuration. We demonstrate that the choice of the piezopolymer transducer technology is suitable for this type of investigation and the advantages of the proposed transducer assembly and bonding are described. A full set-up is described to determine the relationship between the time of flight of the recorded signals and the applied bending moment. Interdigital transducers were designed according to simulations of the dispersion curves, in order to operate at a central frequency of $450 \mathrm{kHz}$. This frequency corresponds to a central wavelength of $16 \mathrm{~mm}$ and to a group velocity of about $6000 \mathrm{~m} / \mathrm{s}$ for the first symmetric guided wave mode. The variations in the time of flight of ultrasonic recorded signals were measured as a function of the variations in the bending moment. The static and dynamic load tests were in good agreement with strain gage measurements performed in the micro deformation range $(0-1400 \mu \mathrm{m} / \mathrm{m})$.
\end{abstract}

\section{Introduction}

Recent developments in the technology of materials have led to an increase in the use of composite laminates, which are employed in aircraft, space vehicles, boats, bridges, building restorations, and many other engineering applications. The key features of both carbon-fiber-reinforced (CFRP) and glass-fiber-reinforced (GFRP) plastic composite laminates are their strength and stiffness properties, which can be tailored to meet the design requirements of CAD tools. In many applications, composites are employed to build parts of complex structures and are subjected to bending and/or tensile stresses. Normal tensile stresses can be monitored by means of strain measurements. Overstresses can lead to the initiation of damage and to the growth of existing voids and small cracks to the point of forming a macroscopic crack. The damage initiation processes are due to local stresses in the fibers and in the reinforcement matrix interface. Hence, it is important to develop monitoring methods for in situ strain and stress measurements of the status of composite materials, including bending stress. For tensile stresses, two nondestructive testing techniques exist that are suitable for experimental investigations: ultrasonic methods $[1,2]$ and fiber Bragg grating [3,4]. Both methods can also be compared with standard strain gage methods for sensitivity, accuracy, and applicability to large composite material structures or components. The transducers technology for generating Lamb waves and their relative advantages are reported in [5].

The generation of selected guided wave modes is of particular importance for enhancing the sensitivity of the measurements with particular mechanical stress conditions. In our experience the flexible transducers made of piezopolymer film with interdigital electrodes are an effective solution for damage and impact detection on aircraft reusable components $[6,7]$. For this application we investigated another important feature of flexible transducers that is the acoustic coupling with removable adhesive tapes. With these types of transducers we studied this particular mechanical loading 
condition because it has not yet been fully investigated in the literature. The experimental set-up was arranged (see Section 2) in order to obtain a good approximation of pure bending loading conditions.

In a previous paper [2], we demonstrated that Young's dynamic modulus of a unidirectional carbon fiber, measured with ultrasonic methods, increased significantly with the tensile stress. The experimental measurements of time of flight (TOF) performed under tensile load were used to derive a relationship between strain and the TOF of the leading symmetric Lamb wave mode $S_{0}$. Stiffening effects were observed by means of TOF observations for both unidirectional and multidirectional CFRP laminates.

The use of ultrasonic Lamb waves for the characterization of a bending stress has significant potential for the Nondestructive Evaluation (NDE) of large-area composite laminates, because they can propagate across a vast distance with acceptable signal attenuation. However, the problem of estimating the bending stress by means of TOF measurements needs to be carefully analyzed, because each composite laminate can sustain multiple Lamb wave modes, the phase velocity of which in turn depends on the transducer operating frequencies and on the laminate-thickness product. Interdigital transducers (IDT) are usually employed, because they make it possible to select specific propagation modes that can propagate efficiently in the composite laminate. The frequency tuning range for Lamb wave modes that we chose was obtained by suitably designing the finger width and pitch [8].

In this study, a $4 \mathrm{~mm}$ thick symmetric cross-ply $\left[0^{\circ} / 90^{\circ}\right]$ CFRP laminate was adopted. The phase and group velocity dispersion curves of the Lamb wave modes were estimated. In Section 2, we report the conditions for obtaining pure bending stress. The modeling of the composite laminate and the calculations of the phase and group velocity diagrams in the frequency domain for the transducer design are reported in Section 3. The model that we developed was based on the transfer matrix method proposed by Adler [9]. In Section 4, we describe the experimental set-up realized for the characterization of the CFRP sample with interdigital piezopolymer transducers, and in Section 5 we compare the results obtained with strain gage measurements mounted on the same sample.

\section{Underlying Theory of Pure Bending Stress in Composite Laminates}

In our work, we assumed a composite laminate that was subjected to pure bending on four points ideally without friction. This condition made it possible to have a portion of the laminate with a constant deformation in order to determine the relationship between the applied bending moment and both the TOF and the strain. We assumed the following:

(i) The laminate thickness was $h$, and the front and back sides were identified by the coordinates $z=h / 2$ and $z=-h / 2$, respectively.

(ii) The thickness $(h)$ was the smallest dimension.

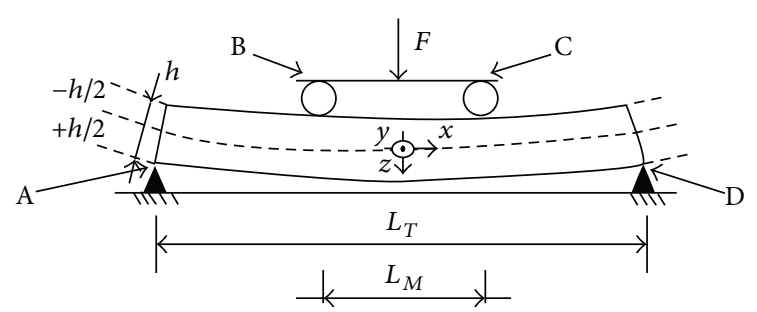

(a)

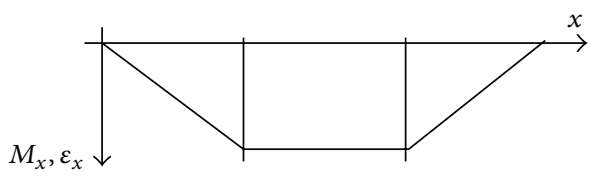

(b)

Figure 1: (a) Pure bending on 4 points (A, B, C, and D) applied to a thin plate: $L_{T}=500 \mathrm{~mm}, L_{M}=265 \mathrm{~mm}$, and $h=4 \mathrm{~mm}$ and $F$ is the applied force in $z$ direction; (b) bending moment $M_{x}$ and longitudinal deformation $\varepsilon_{x}$. We assumed $\varepsilon_{x}$ as being proportional to $M_{x}$.

(iii) No stress and strain existed along $z$ direction.

(iv) $M_{z}$ and $M_{y}$ bending moments along $z$ and $y$ directions, respectively, were equal to zero.

By considering the theory of the propagation of planar waves in a thin laminate we could define three leading equations referring to the three Cartesian orthogonal directions (see Figure 1(a)):

$$
\begin{aligned}
& \frac{\partial \sigma_{x}}{\partial x}+\frac{\partial \tau_{y x}}{\partial y}+\frac{\partial \tau_{z x}}{\partial z}+\rho \cdot f_{x}=\rho \cdot \frac{\partial^{2} u}{\partial t^{2}}, \\
& \frac{\partial \tau_{x y}}{\partial x}+\frac{\partial \sigma_{y}}{\partial y}+\frac{\partial \tau_{z y}}{\partial z}+\rho \cdot f_{y}=\rho \cdot \frac{\partial^{2} v}{\partial t^{2}}, \\
& \frac{\partial \tau_{x z}}{\partial x}+\frac{\partial \tau_{y z}}{\partial y}+\frac{\partial \sigma_{z}}{\partial z}+\rho \cdot f_{z}=\rho \cdot \frac{\partial^{2} w}{\partial t^{2}},
\end{aligned}
$$

where $\rho$ was the density of the laminate, $f_{x}, f_{y}$, and $f_{z}$ were the forces applied in $x, y, z$ directions, $u$, $v$, and $w$ were the displacement vectors, and $\tau_{i j}$ and $\sigma_{k}$ were the shear and the longitudinal stresses, respectively.

The force considered was only applied in $z$ direction, indicated as $F$ (see Figure 1(a)). The resulting forces applied in two points (B and $\mathrm{C}$ ) on the laminate were equal to $F / 2$ and the only bending moment, $M_{x}$, was along $x$-axis with the distribution reported in Figure 1(b).

Conditions (i), (ii), and (iii) led to the following conditions in the stress components:

$$
\tau_{x z}=\tau_{z x}=\tau_{y z}=\tau_{z y}=\sigma_{z}=0 .
$$

Based on previous assumptions, the shear strains $\gamma_{x y}$ and the longitudinal strain $\varepsilon_{y}$ could be considered zero, and the resulting strain $\varepsilon_{x}$ was only in the longitudinal direction. Within a small deformations range, $\varepsilon_{x}$ was proportional to the bending moment $M_{x}$. It was observed that the bending 


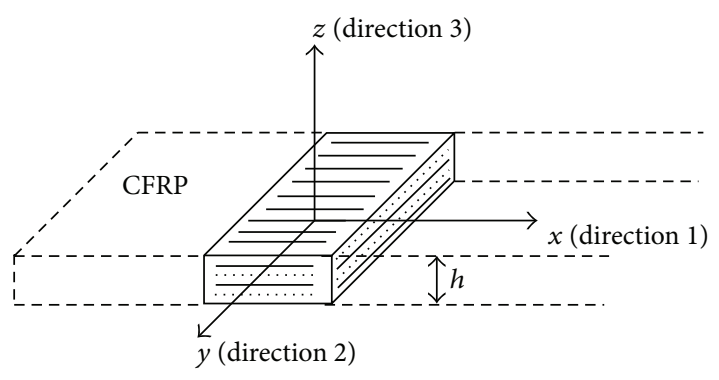

FIGURE 2: Reference system adopted for guided mode analysis of the CFRP cross-ply $\left[0^{\circ} / 90^{\circ}\right]$ laminate.

moment $M_{x}$ and the longitudinal strain $\varepsilon_{x}$ were constant between the two load application points $\mathrm{B}$ and $\mathrm{C}$ (within $L_{M}$ segment). Within this region, by considering $L_{T}$ as the distance between $\mathrm{A}$ and $\mathrm{D}$ points, the ideally bending moment $M_{x}$ was constant and equal to $\left(L_{T}-L_{M}\right) \times F / 4$. Based on these assumptions (four points bending without friction) our experiments were carried out on pure bending stress measurements with ultrasonic TOF and strain gage methods using only one direction for the measurements.

\section{Interdigital Transducer Design by Group Velocity Analysis for a CFRP Cross-Ply $\left[0^{\circ} / 90^{\circ}\right]$ Laminate}

A 4 mm thick CFRP cross-ply $\left[0^{\circ} / 90^{\circ}\right]$ sample was considered in order to calculate phase and group velocity dispersion curves of ultrasonic guided waves. The reference system for modes calculation is shown in Figure 2.

We calculated phase and group velocity dispersion curves for the longitudinal guided wave propagation in our crossply $\left[0^{\circ} / 90^{\circ}\right]$ CFRP laminate (see Figures $3(\mathrm{a})$ and $3(\mathrm{~b})$ ) by developing a dispersive modes calculation program in MATLAB. The shear horizontal $(\mathrm{SH})$ and vertical (SV) modes were not considered in the computation for the configuration adopted in the TOF measurements. To verify the accuracy of the developed model, we first compared the analytical solutions of phase and group velocity dispersion curves of the homogeneous materials: aluminum, copper, and nickel. Afterwards, we compared the dispersion curves of both unidirectional and cross-ply $\left[0^{\circ} / 90^{\circ}\right]$ composite materials obtained with our model, as compared to those reported in the literature for a similar composite material [10].

The use of TOF measurements in order to characterize the pure bending stress of composite material led us to consider group velocity measurements of the received signals in correspondence with different bending conditions. Analysis of the TOF measurements can be carried out by using the cross-correlation method or by means of an estimate of the delays in the signals at $50 \%$ of the maximum of their envelope maximum amplitudes. Preliminary measurements performed on the composite material sample in stress-free conditions showed that the cross-correlation method was much more sensitive to the TOF variations.
TABLE 1: IDTs design parameters: width $(W)$, length $(L)$, fingers separation $\left(S=\lambda_{\mathrm{IDT}} / 2\right)$, number of fingers $(N), \varepsilon_{\mathrm{IDTmin}}=\lambda_{\mathrm{IDT}}-W$, and $\lambda_{\mathrm{IDTmax}}=\lambda_{\mathrm{IDT}}+W$.

\begin{tabular}{cccccc}
\hline$W[\mathrm{~mm}]$ & $L[\mathrm{~mm}]$ & $\lambda_{\text {IDT }}[\mathrm{mm}]$ & $\lambda_{\text {IDTmin }}[\mathrm{mm}]$ & $\lambda_{\text {IDTmax }}[\mathrm{mm}]$ & $N$ \\
\hline 3 & 27.5 & 16 & 12 & 18 & 6
\end{tabular}

3.1. Mode Excitation by means of an Interdigital Transducer. We used a pair of interdigital transducers in pitch-catch configuration in order to transmit and receive the Lamb wave modes [11].

Considering interdigital transducers we can define the following relationship:

$$
\frac{v_{p}}{f_{c} \times h}=\frac{\lambda_{\mathrm{IDT}}}{h}
$$

where $v_{p}$ is the phase velocity of the ultrasonic wave, $f_{c}$ is the central frequency for the driving signal, $h$ is the thickness of the CFRP cross-ply $\left[0^{\circ} / 90^{\circ}\right]$ sample, and $\lambda_{\text {IDT }}$ is the wavelength of the interdigital transducer. In our case study $\lambda_{\text {IDT }}=16 \mathrm{~mm}$ (see Table 1), the operating frequency $f_{c}$ is $450 \mathrm{kHz}$, and $h=4 \mathrm{~mm}$ (see Figure 3(a)).

Furthermore, the dimension of the transducer finger can be chosen to cover a range of wavelengths that in our case are $\lambda_{\text {IDTmin }}=390 \mathrm{kHz}$ and $\lambda_{\text {IDTmax }}=550 \mathrm{kHz}$. The designed transducer will operate in the range of corresponding frequencies between these two values, with the advantage of the selection of two quasi-non-dispersive modes $A_{1}$ and $S_{1}$. In particular, considering Figure 3(b), the central frequency for the driving signal $f_{c}=450 \mathrm{kHz}$ corresponds to a group velocity of about $6000 \mathrm{~m} / \mathrm{s}$. The group velocity of these two modes is very close and so we have verified this characteristic experimentally by applying the cross-correlation method between two signals acquired at two different distances from the IDT received for the calculation of the group velocity. The measurements were carried out on the same composite material in stress-free conditions and using the preliminary set-up reported in [12]. The number of cycles of the burst driving signal at central frequency $f_{c}$ was experimentally evaluated. A similar approach is reported in [13].

Figure 4 shows a typical signal detected in a stress-free path on the $4 \mathrm{~mm}$ thick CFRP composite in correspondence with a distance of $160 \mathrm{~mm}$ between the transmitting and the receiving IDTs. We can observe that the two modes $A_{1}$ and $S_{1}$ overlap and are not distinguishable in the wavelet. The driving signal, which is characterized by four cycles of sine wave at $f_{c}=450 \mathrm{kHz}$, is also shown in the same figure.

\subsection{Piezopolymer IDTs Coupling on CFRP Laminate. An} important phase of the experimental set-up preparation is the coupling of the piezopolymer transducers on the CFRP laminate. The standard solution for piezoceramic ultrasonic transducers with a rigid case is the acoustic coupling gel; this method has been tested also with our flexible IDTs but does not guarantee repeatable measurements because of the inhomogeneous layer underneath the large transducer surface $(25 \mathrm{~mm} \times 40 \mathrm{~mm})$ and the lack of keeping the position due to the change of adhesion force due to temperature 


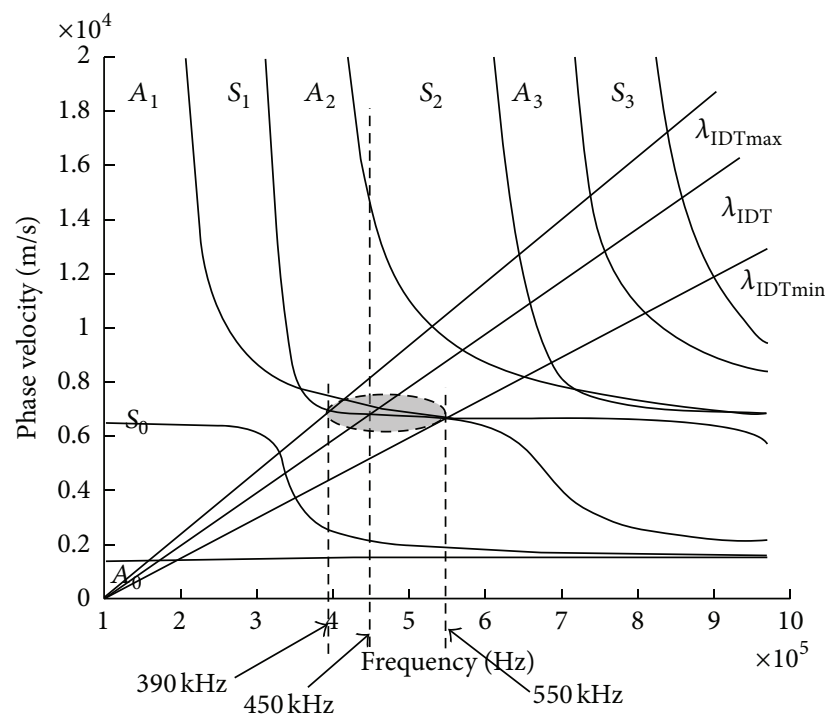

(a)

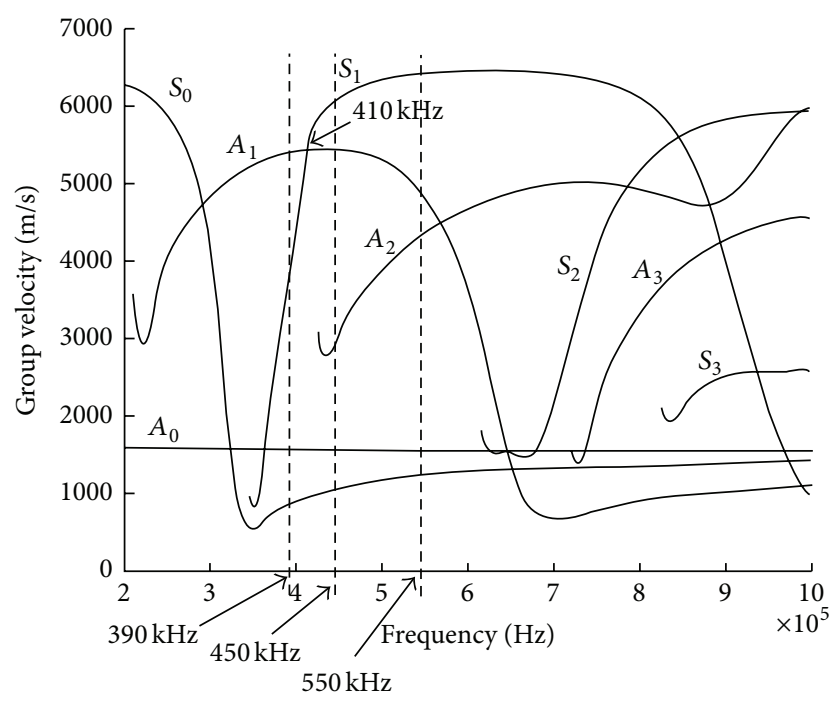

(b)

FIgure 3: (a) Phase velocity dispersion curves for a $4 \mathrm{~mm}$ thick CFRP cross-ply $\left[0^{\circ} / 90^{\circ}\right]$ laminate with superimposing our interdigital transducer characteristics and operating frequency; (b) group velocity dispersion curves for a $4 \mathrm{~mm}$ thick CFRP cross-ply $\left[0^{\circ} / 90^{\circ}\right]$ laminate.

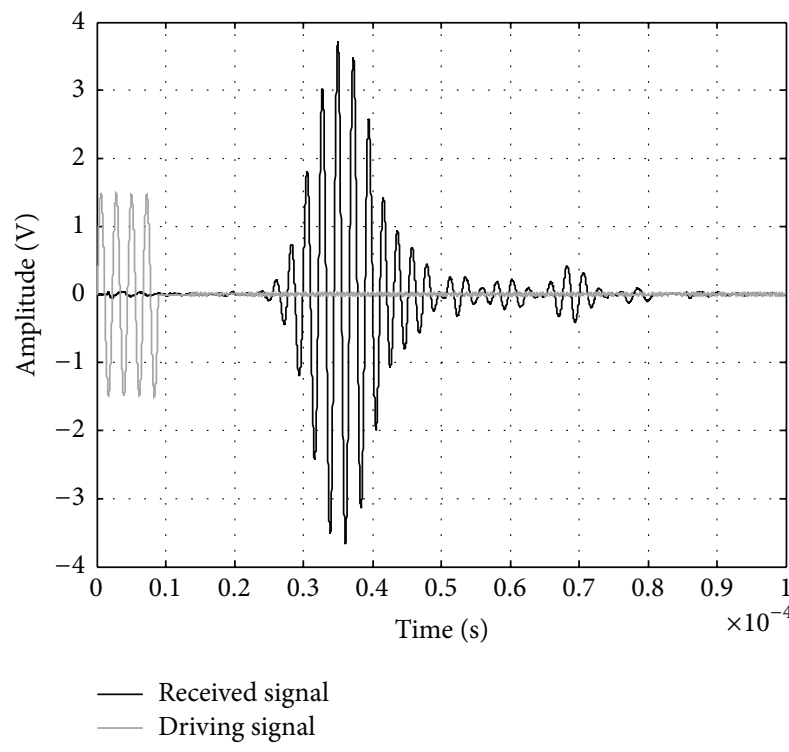

FIGURE 4: Driving signal (light grey) and received signal (black) detected in a stress-free path on the $4 \mathrm{~mm}$ thick CFRP composite, when considering a distance of $160 \mathrm{~mm}$ between the transmitting and the receiving IDT.

variation of the acoustic coupling gel and drying. Then a more repeatable and acoustically efficient solution is the permanent fixing by bonding the transducer to the CFRP by selecting a suitable epoxy adhesive. The bonding procedure, with a standard epoxy adhesive, has been carried out at environmental conditions (the curing takes 12 hours) because the curing process at higher temperatures $\left(>80^{\circ} \mathrm{C}\right)$ may lose the piezoelectric effect on piezopolymer transducers. This method has been compared to an alternative method presented in this work based on a removable solution with bonding with adhesive tape. This novel method has a great advantage that does not affect permanently the surface of the sample under test and makes the eventual transducers replacement easy; moreover during experimentation the transducers orientation and positioning can be changed to obtain optimized characteristics of the ultrasonic guided wave propagation. However the removable solution requires the insertion of an additional layer between the piezopolymer film and the surface that can alter the acoustic coupling of the length extensional deformation of the piezopolymer material. A comparison of these two solutions has been carried out and described in this section.

The first solution has been implemented by carefully deploying the epoxy on a layer with thickness similar to that of the piezopolymer film $(100 \mu \mathrm{m})$.

In Figures 5(a)-5(b) and 6(a)-6(b) are shown the two phases of the bonding on a unidirectional CFRP sample of an IDT. The solution adopted for the connections of the IDT to the coaxial cable is the use of PCB technology with pressure that ensures low contact resistance between copper pad of the PCBs and gold metallization of the electrodes; the pressure is obtained by micro rivets. The IDT is aligned along the carbon-fiber direction.

The bonding of the IDTs biadhesive tape is carried out by selecting different types and choosing the one that has lower compliance and large temperature range for maintaining adhesion force.

In practice we select the biadhesive tape model Eurocell with thickness $28 \mu \mathrm{m}$ with acoustic impedance that best matches the coupling between the piezopolymer film and the CFRP. This technological study demonstrates that coupling with biadhesive makes IDTs reusable with respect to standard epoxy adhesive [12]. 


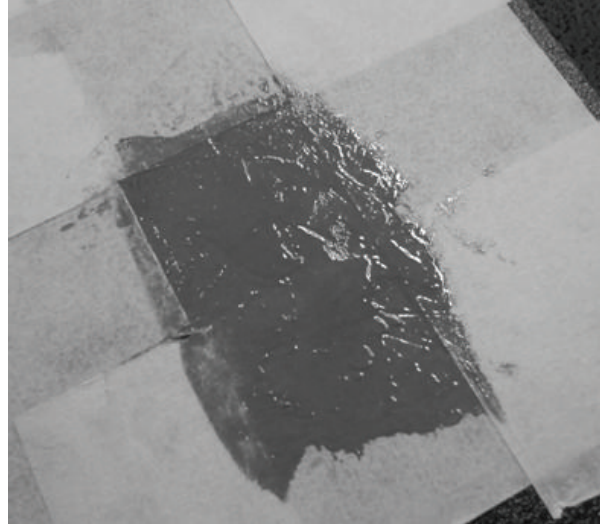

(a)

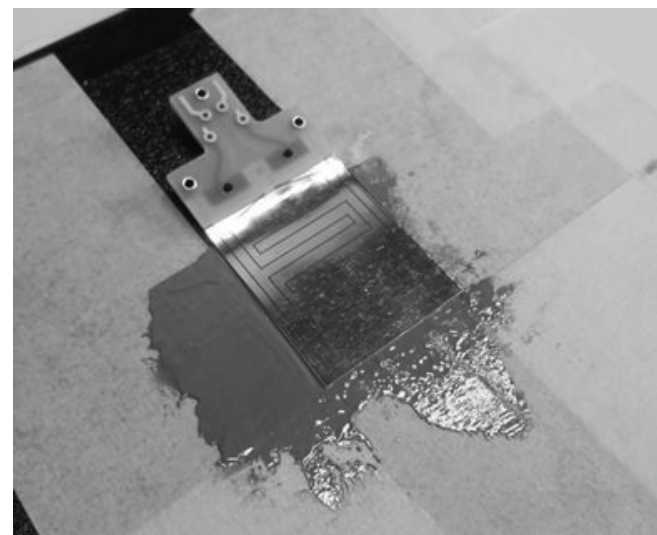

(b)

FIgURE 5: (a) Phase one of the bonding on a unidirectional CFRP sample of an IDT. (b) Phase two of the bonding on a unidirectional CFRP sample of an IDT.

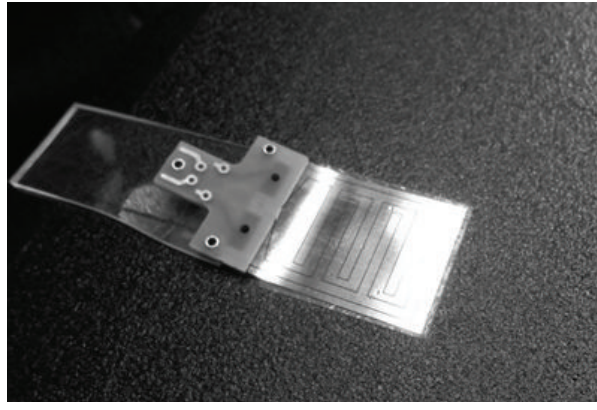

(a)

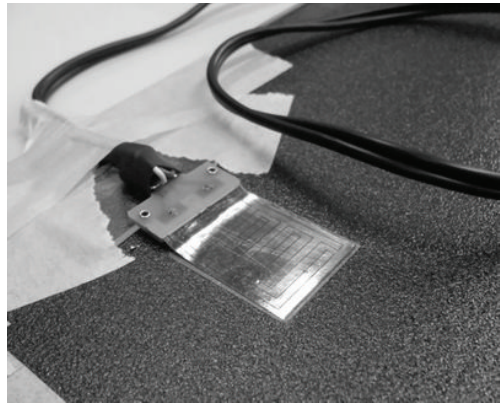

(b)

FIgURE 6: (a) Particular of the IDT bonded on a unidirectional CFRP. (b) Particular of the IDT bonded on a unidirectional CFRP with cable.

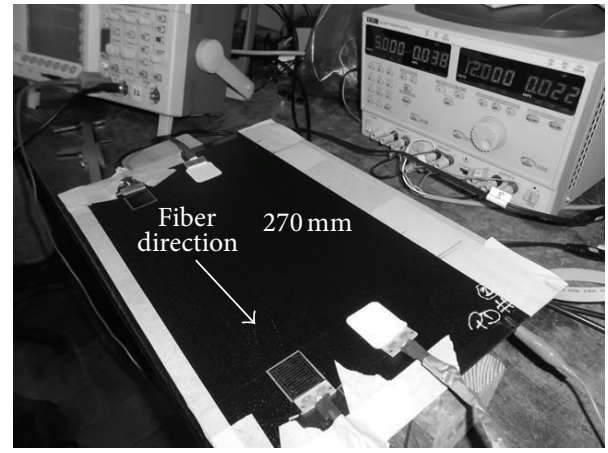

FIGURE 7: Two pairs of piezopolymer IDTs at the same distance of $270 \mathrm{~mm}$ in pitch-catch configuration.

These two transducers have been also sealed with a thin white adhesive patch to be protected from the environment. This soft patch does not change the acoustic response of the transducers.

For the comparison of the two solutions we mounted two pairs of piezopolymer IDTs at the same distance of $270 \mathrm{~mm}$ in pitch-catch configuration, as shown in Figure 7.
The front-end electronics drives the two transmitting transducers with a burst of 4 cycles at the frequency of $180 \mathrm{kHz}$ through an $\mathrm{H}$ bridge power amplifier with amplitude $96 \mathrm{~V}_{\mathrm{pp}}$. The low noise amplifier has a gain of $60 \mathrm{~dB}$ and $-3 \mathrm{~dB}$ bandwidth of $500 \mathrm{kHz}$. At this frequency $A_{0}$ mode is also propagated.

In Figure 8 are reported the two received signals propagated along paths A-A and B-B shown in Figure 7.

The comparison points out that the permanent bonding with epoxy provides a better acoustic coupling of about $+6 \mathrm{~dB}$ and the removable solution does not alter the frequency content of the ultrasonic signal and guarantees an adequate signal-to-noise ratio. The small delay between the two signals is due to the different length of paths A-A and B-B.

\section{Experimental Set-Up}

For our experiments, we devised an experimental set-up consisting of five main items:

(i) A CFRP sample.

(ii) A mechanical set-up for pure bending stress application. 


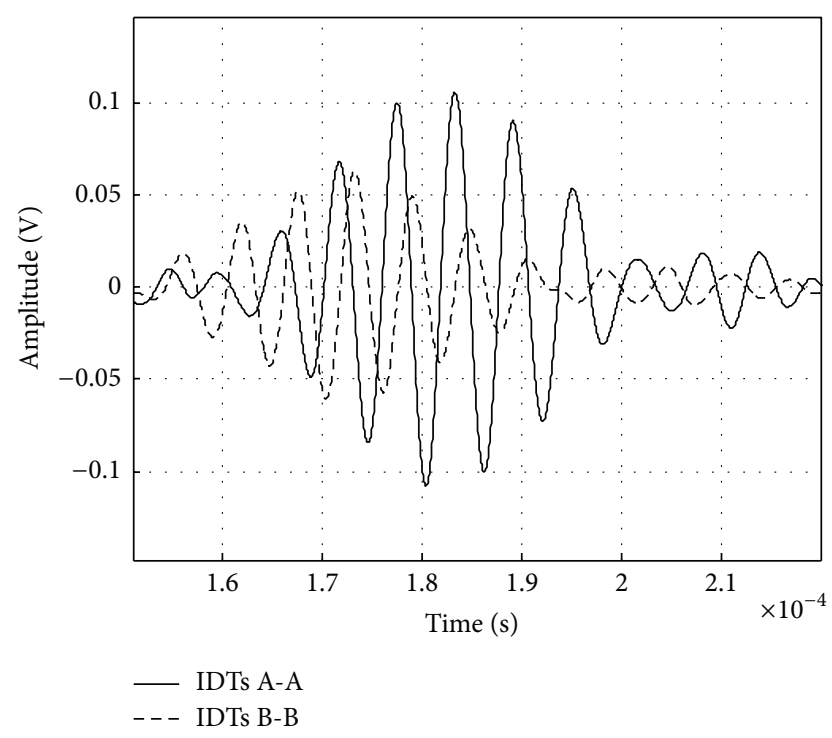

FIgURE 8: The two received signals propagated along the paths A-A and B-B shown in Figure 7.

(iii) An electronic set-up for the IDTs' signal excitation/acquisition and signal processing.

(iv) A pair of piezopolymer IDTs.

(v) A strain gage meter.

The CFRP sample was a $4 \mathrm{~mm}$ thick cross-ply $\left[0^{\circ} / 90^{\circ}\right]$ laminate. The area of the CFRP sample $\left(176 \times 500 \mathrm{~mm}^{2}\right)$ was large enough to place the IDTs in pitch and catch configuration with a direct path length, $L_{0}=160 \mathrm{~mm}$. Two gages were placed outside the ultrasonic wave's transmission path, in order to measure the longitudinal and traversal surface strain (see Figure 5). The transversal gage was included in order to verify that the actual deformation along this direction was negligible, as was assumed in Section 2. Moreover, a gage for compensating the temperature gradients was mounted on a small piece of the same CFRP material and was placed close to the other gage bonded on the CFRP sample surface.

The experimental set-up for the application of pure bending stress is reported in Figure 9.

The mechanical set-up for generating pure bending stress shown in Figure 10 consisted of a metallic structure containing two rigid aluminum bars placed at a relative distance, $L_{T}=500 \mathrm{~mm}$, for holding the CFRP laminate, which was suspended at a certain height, $h_{M}=60 \mathrm{~mm}$, from the bench plane. Force was applied to the CFRP laminate by means of two steel cylinders assumed to be perfectly rigid, each with a diameter of $15 \mathrm{~mm}$. The two cylinders were placed at a distance $L_{M}=265 \mathrm{~mm}$. Force $F$ was applied perpendicular to the plane of the laminate in the central part of the area delimited by $L_{M}$ (see Figure 10). To approximate a point-like application, we employed a bolt with a pin-ball termination. By screwing the bolt, the force was progressively applied, causing the CFRP sample being tested to bend. This bending developed mainly along $x-z$ cross section of the laminate and led to an almost constant deformation on the CFRP sample within $L_{M}$.

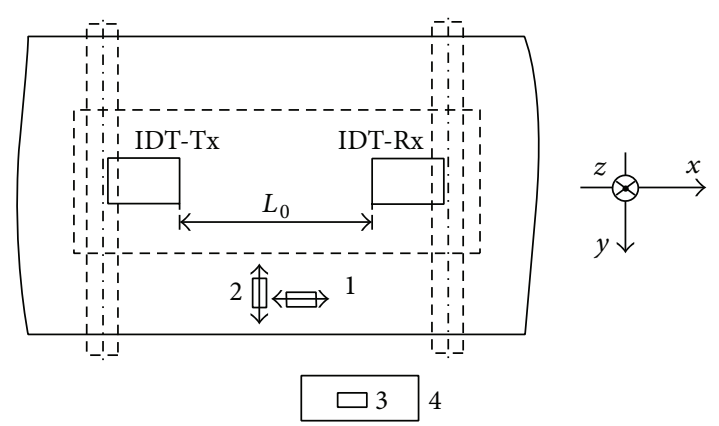

(a)

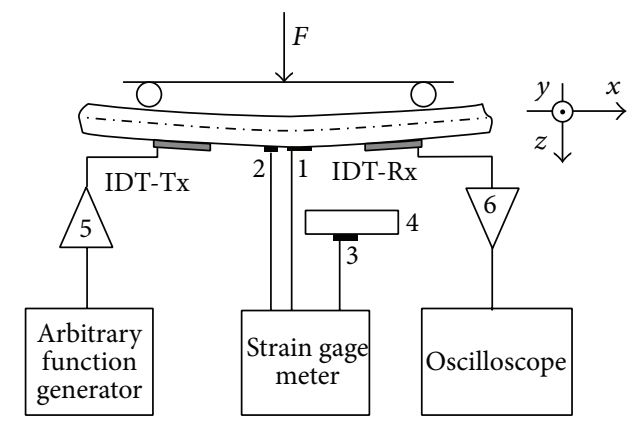

(b)

FIgURE 9: Experimental set-up; (a) bottom view of sample with the sensors; (b) cross section of sample; 1: longitudinal gage, 2 : transversal gage, 3: gage used to compensate the thermal drift, 4: CFRP stress-free sample, 5: power amplifier, and 6: low noise amplifier.

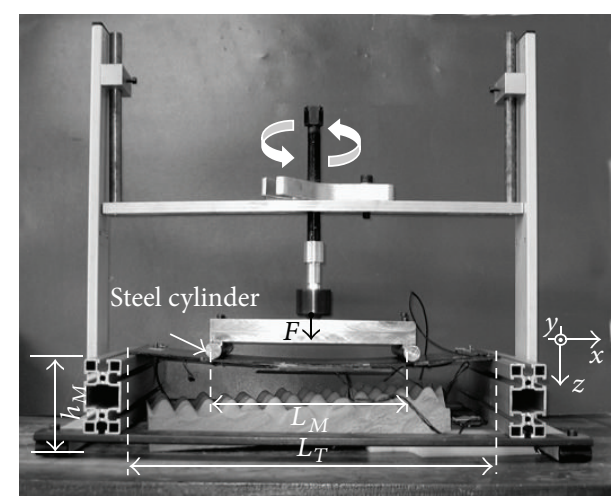

FIGURE 10: Picture of the mechanical/experimental set-up for pure bending stress application on 4 points; $h_{M}=60 \mathrm{~mm}, L_{M}=265 \mathrm{~mm}$, and $L_{T}=500 \mathrm{~mm}$.

For the signals acquisition, we used a TEKTRONIX model TDS 3013b digital oscilloscope with a 9-bit resolution and 2 ns sampling time. The driving burst was generated by an AGILENT model $33220-20 \mathrm{MHz}$ arbitrary function generator, which was connected to a customized linear power amplifier (gain $45 \mathrm{~dB},-3 \mathrm{~dB}$ bandwidth $80 \mathrm{kHz}-1 \mathrm{MHz}$ ) for driving the transmitting transducer. The excitation signal was a burst with four sine cycles with amplitude $1.5 \mathrm{~V}_{\mathrm{pp}}$ and frequency $450 \mathrm{kHz}$, which resulted in a peak-to-peak voltage of $267 \mathrm{~V}$ applied to the transmitting transducer. 


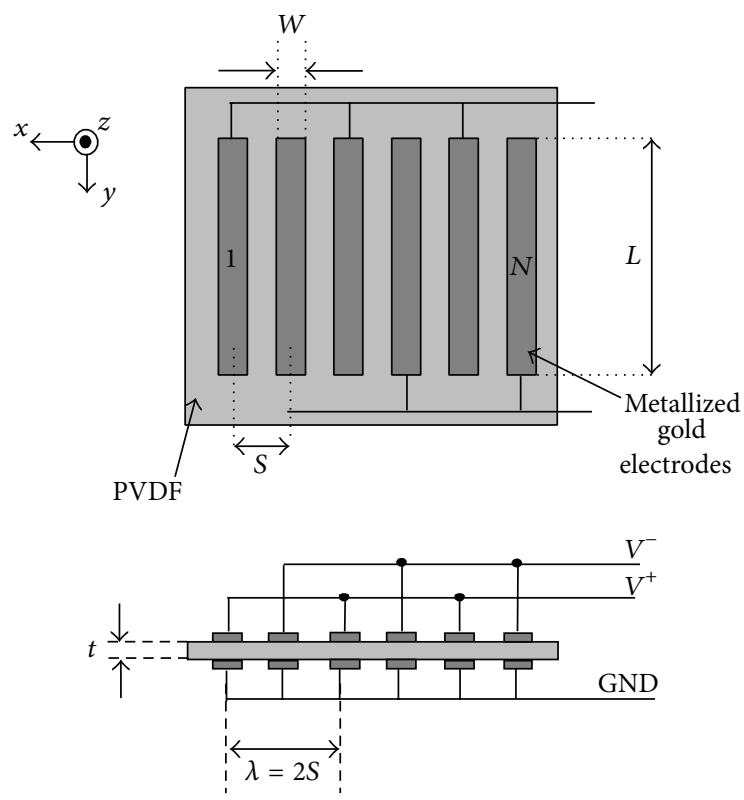

FIGURE 11: Geometry of interdigital transducers of gold metallized electrodes on a piezopolymer film (drawing not in scale) with characteristics reported in Table 1.

The receiving transducer was connected to a low noise instrumentation amplifier with $-3 \mathrm{~dB}$ bandwidth of $1 \mathrm{MHz}$ and voltage gain of $56 \mathrm{~dB}$. This preamplifier was designed to match the piezopolymer electrical impedance for obtaining a high signal-to-noise ratio. The digitized signals were then transferred to a PC and processed offline.

Two piezopolymer IDTs with design parameters reported in Table 1 were used in a pitch-catch configuration placed on the CFRP, as shown in Figure 10. The material used for the sensor design was a commercial copolymer P(VDFTrFE) film (PiezoTech S.A., St. Louis, France) with thickness $t=100 \mu \mathrm{m}$ and a gold metallization with an approximate thickness of $0.1 \mu \mathrm{m}$, mass density $\rho_{m}=1780 \mathrm{~kg} / \mathrm{m}$, and longitudinal velocity $V_{\mathrm{PVDF}}=2200 \mathrm{~m} / \mathrm{s}$.

The IDT electrode geometry, which is shown in Figure 11, was transferred onto the metallized piezopolymer film by means of a patented laser marking process [14].

An interlaced electrode configuration was adopted. This transducer configuration was differential, because the two series of electrodes (also called fingers) were driven with opposite phase signals $(V+$ and $V-$ ) and had the same reference ground electrode (GND). The basic transducer design parameters consisted of the width $(W)$, length $(L)$, piezopolymer film thickness $(t)$, separation of the fingers $(S)$, and the number of fingers $(N)$. The interlaced configuration requires that finger separation $S$ should be half of the wavelength $\lambda_{\text {IDT }}$ relative to the guided wave mode selected. Parameter $W$ changes the effective IDT area and influences the acoustic response in the wave number domain. Length $L$ defines the directivity of the transducers according to the approximated relationship for laminates [9].

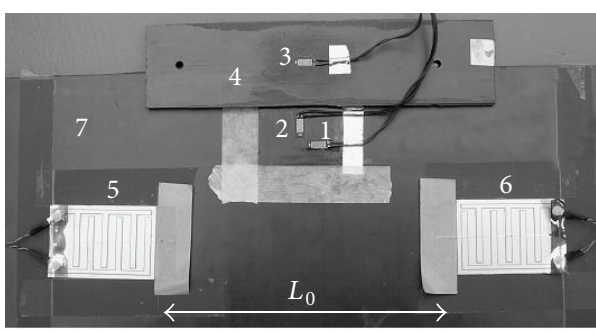

FIGURE 12: Zoom on sensors applied to sample of CFRP. 1: longitudinal gage, 2: transversal gage, 3: gage used to compensate the thermal drift of the other two, 4: portion of the same CFRP sample used for compensation gage without mechanical stress, 5: IDT for signal transmission (Tx-IDT), 6: IDT for signal detection (Rx-IDT), and 7: CFRP sample.

The surface deformation was measured by a VISHAY model P-3500 strain gage meter that has the following characteristics:

(i) Resistance @ $24^{\circ} \mathrm{C}: 350.0 \Omega \pm 0.15 \%$.

(ii) Gage factor @ $24^{\circ} \mathrm{C}: 2.095 \pm 0.5 \%$.

(iii) Transversal sensitivity coefficient $\left(k_{t}\right):+0.1 \pm 0.2 \%$.

Two strain gages, bonded on the CFRP sample in longitudinal and transversal directions, are shown in Figure 12. The third one was used to compensate the thermal drift of the other two. The longitudinal and transversal surface strains were read on a 5-digit display with $1 \mu \varepsilon=1 \mu \mathrm{m} / \mathrm{m}$ resolution.

With the longitudinal gage, we measured deformation along $x$ direction $\left(\varepsilon_{x}\right)$; instead, with the transversal gage, we measured deformation along $y$ direction $\left(\varepsilon_{y}\right)$. Since preliminary measurements confirmed that the deformation along $y$ direction was two orders of magnitude lower than the deformation in $x$ direction, in the tests reported in Section 5 we considered only the longitudinal deformation. On the front $(z=-h / 2)$ and back faces $(z=+h / 2), \varepsilon_{x}$ assumed the values $-\varepsilon_{B}$ and $+\varepsilon_{B}$, respectively. For the longitudinal gage shown in Figure 14, we considered $+\varepsilon_{B}$ value. $\varepsilon_{x}$ values were those used when measuring the bending status of a composite laminate by means of a gage bonded on the surface.

\section{Results with Static and Dynamic Loads}

Using the set-up described in Section 4, we carried out several tests in different pure bending loading conditions. Our preliminary measurements involved some signals acquired in stress free bending conditions and in medium and maximum bending conditions. Three acquired signals are shown in Figure 13. The time window was placed on the first seven peaks of the received signals. The propagating signal was a sine burst of four cycles at frequency $f_{c}=450 \mathrm{kHz}$. In Figure 13, we show that the envelopes of the three signals were equal in a time window of $20 \mu \mathrm{s}$.

From the results of these preliminary tests, we succeeded in establishing the following measurement protocol in order to have repeatable measurements:

(i) Warm-up of the instruments at a constant room temperature, for 30 minutes. 


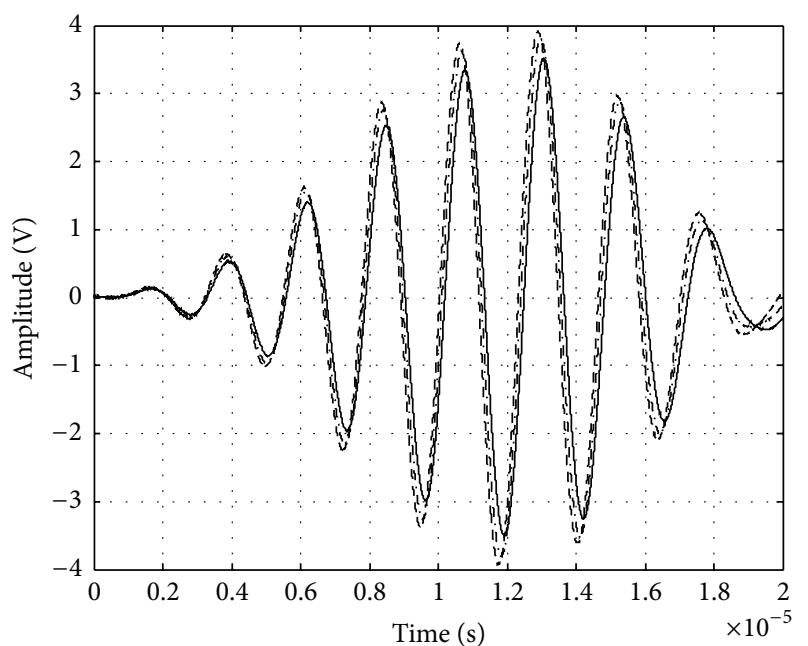

— Signal stress-free bending conditions

- . - Signal medium bending conditions

- - - Signal maximum bending conditions

Figure 13: Three signals acquired in medium and maximum bending conditions and in stress-free conditions. The $20 \mu$ s time window was placed on the first seven peaks of the received signals.

(ii) Calibration, definition of the gage factor, and balancing the Wheatstone bridge of the strain gage meter.

(iii) Loading and unloading the composite laminate for $3 / 4$ bending full cycles.

(iv) Acquisition of the reference signal from Rx-IDT in stress-free condition.

(v) Acquisition of the first 7 peaks of the signals received in a time window of $20 \mu$ s at different bending positions, such as those reported in Figure 13.

The TOF variation $(\Delta t)$ was calculated using the crosscorrelation between pairs of acquired signals at different loads using a script based on the MATLAB cross-correlation function "xcorr": In particular all received signals in a time window of $20 \mu$ s at different bending positions (see, e.g., signals shown in Figure 13) were cross-correlated with the reference signal from Rx-IDT in stress-free condition and the resulting TOF variation values were recorded.

5.1. Static Load Test with Assigned Values of Force. In this experiment, we applied known forces, $F$, by means of castiron weights increased from 0 to $300 \mathrm{~N}$; for each force value, the surface strain $\varepsilon_{B}$ and TOF variation $(\Delta t)$ were collected in static conditions, after a rest period of $60 \mathrm{~s}$. Five data series were collected.

The data plots for every series and for the corresponding linear fitting are shown in Figure 14. The continuous lines represent the linear fitting expressed by

$$
\begin{aligned}
& \varepsilon_{B} \approx 1.80 \cdot F+5.9, \\
& \Delta t \approx-0.20 \cdot F+0.8 .
\end{aligned}
$$

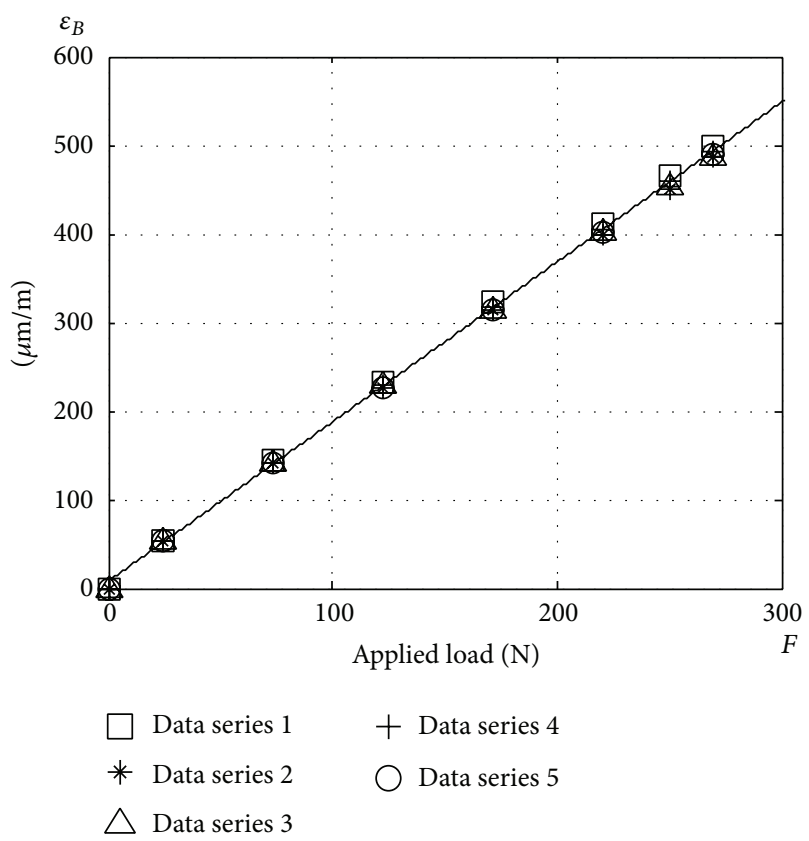

(a)

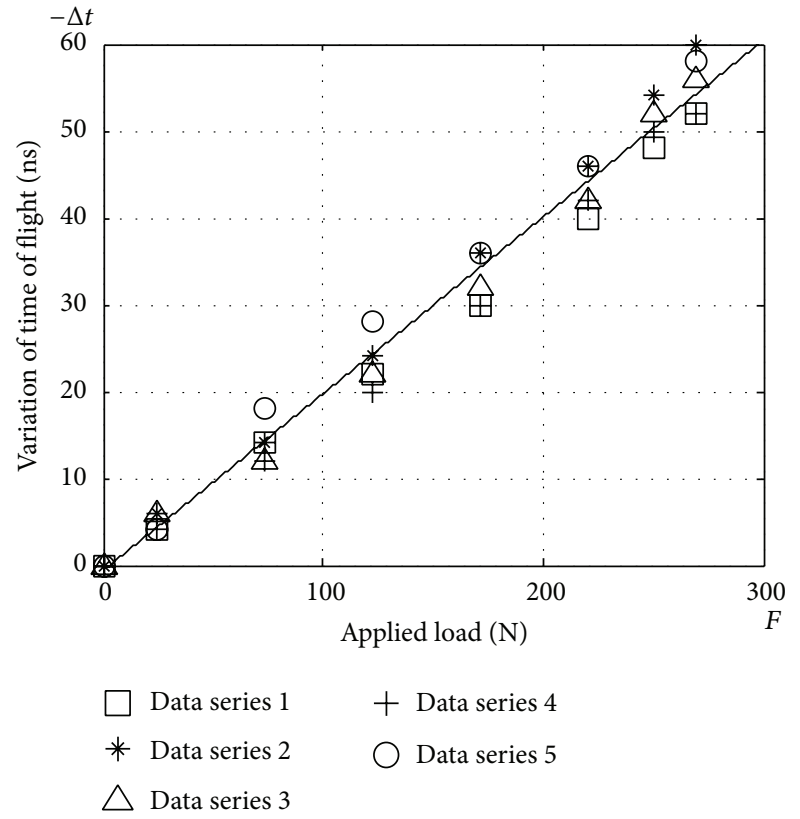

(b)

FIgURE 14: Five series of data acquired with assigned loads in static conditions. The surface deformation versus applied load is reported on (a), while the variation of TOF versus applied load is reported on (b).

The resulting standard deviations were $\sigma_{\varepsilon}=4.5 \mu \varepsilon$ and $\sigma_{\Delta t}=$ $2.5 \mathrm{~ns}$.

5.2. Dynamic Load Tests. During these experiments, we applied an increasing bending load monotonically by slowly screwing the bolt in a continuous way. We then measured the surface strain $\varepsilon_{B}$ and TOF variation $(\Delta t)$ simultaneously. 


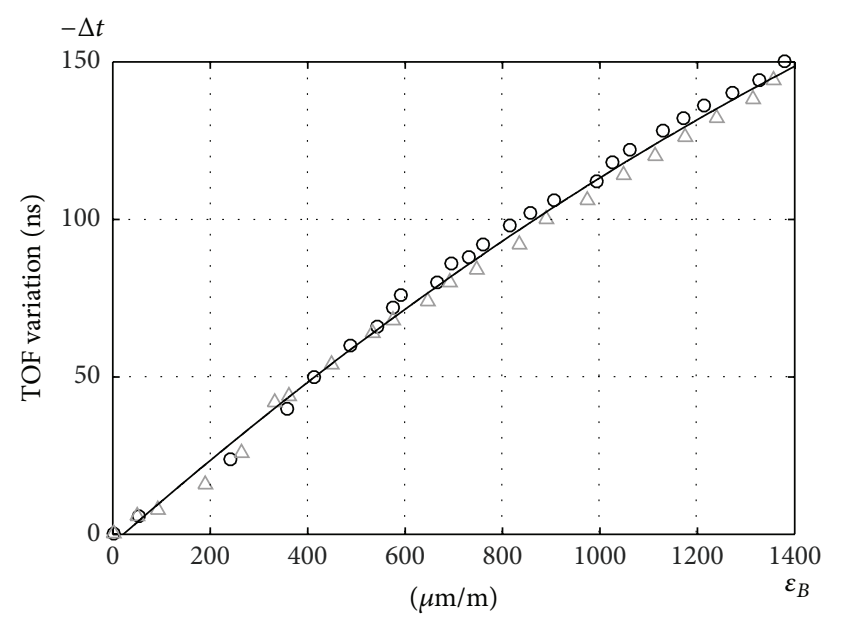

○ Data series 1

$\triangle$ Data series 2

Figure 15: Two series of data acquired in dynamic conditions. The continuous line indicates the quadratic fitting.

The ultrasonic signals and strain gage measurements were recorded for each load value. The dynamic test for the full load application lasted for 4 minutes. The time lag between the ultrasonic signal and the strain gage acquisitions, which was due to the setting time of the strain gage meter display output, was less than $9 \mathrm{~s}$.

In these experimental conditions, we plotted $\Delta t$ values versus the surface strain $\varepsilon_{B}$. The two data series and the corresponding quadratic fits are reported in Figure 15.

The quadratic fitting curve, which is expressed in [ns] for $\Delta t$ and in $[\mu \varepsilon]$ for $\varepsilon_{B}$, was evaluated on $\varepsilon_{B}$ range, which extends from 0 to $1400 \mu \mathrm{m} / \mathrm{m}$. The continuous line is the quadratic fit of two data series. It was found to be

$$
\Delta t \approx 2 \cdot 10^{-5} \cdot \varepsilon_{B}^{2}-0.14 \cdot \varepsilon_{B}+2.9
$$

with a standard deviation of $2.9 \mathrm{~ns}$.

5.3. Static Load Tests. Similar to the previous experiments we measured the surface strain $\varepsilon_{B}$ and $\Delta t$ in static load conditions over $\varepsilon_{B}$ range extending from 0 to $1400 \mu \mathrm{m} / \mathrm{m}$. The relative plotting of eight data series is reported in Figure 16.

The quadratic fit in [ns] for $\Delta t$ and in $[\mu \varepsilon]$ for $\varepsilon_{B}$ is reported in Figure 16 as a continuous line. It was expressed as

$$
\Delta t \approx 1.6 \cdot 10^{-5} \cdot \varepsilon_{B}^{2}-0.12 \cdot \varepsilon_{B}+1.6
$$

with an estimated standard deviation of $3.6 \mathrm{~ns}$.

In this case, the standard deviation was greater than the one obtained in the dynamic test, probably due to the effect of a mechanical hysteresis that developed thanks to the effect of eight successive static load applications.

5.4. Static Loading/Unloading Tests. These tests were performed with a series of four repetitive loading and unloading cycles of the laminate. The measurement procedure was the same as that of the previous tests.

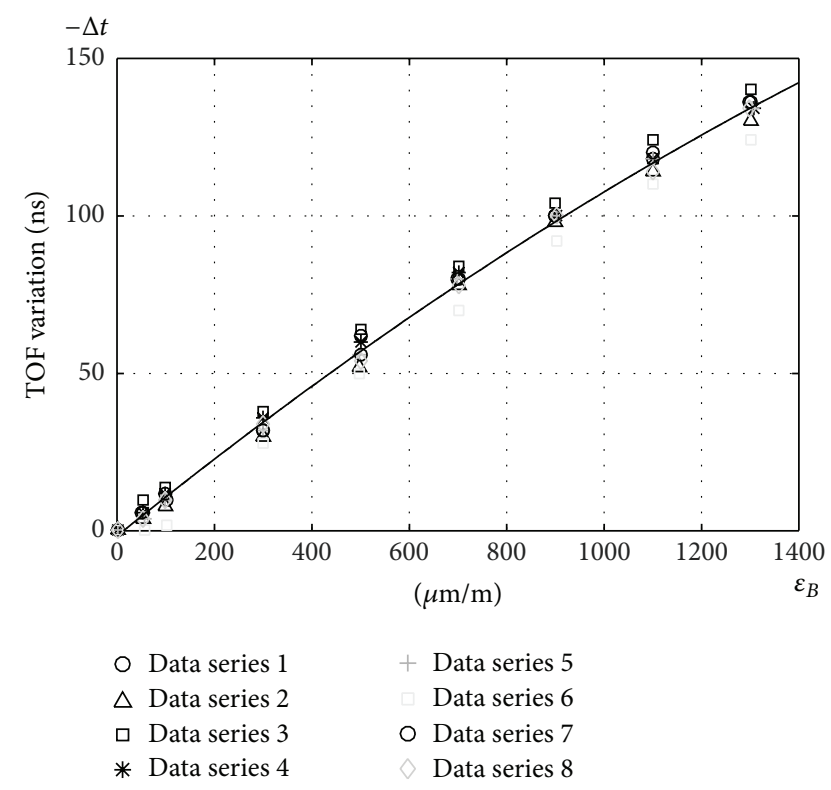

FIGURE 16: Eight series of data acquired in static conditions. The continuous line indicates the quadratic fitting.

The loading and unloading measurement results are shown, respectively, in Figures 17(a) and 17(b). Similar to the previous tests, we also found a good fit for this micro deformation in two quadratic regressions:

$$
\begin{gathered}
\text { Loading } \Longrightarrow \Delta t=2.0 \cdot 10^{-5} \cdot \varepsilon_{B}^{2}-0.13 \cdot \varepsilon_{B}+0.7, \\
\text { Unloading } \Longrightarrow \Delta t=2.3 \cdot 10^{-5} \cdot \varepsilon_{B}^{2}-0.14 \cdot \varepsilon_{B}+4.6 .
\end{gathered}
$$

The estimated standard deviations were $3.1 \mathrm{~ns}$ and $3.7 \mathrm{~ns}$ for (7) and (8), respectively.

\section{Conclusions}

The work has demonstrated that piezopolymer transducers are suitable to select guided waves mode for the measurements of TOF variation related to pure bending stress in composite materials. By experimental characterization or simulation of Lamb waves in the composite laminate, it is possible to design piezopolymer IDTs operating with tuned central frequency and bandwidth. In the specific test sample we designed and fabricated piezopolymer interdigital transducers of $\lambda_{\text {IDT }}=16 \mathrm{~mm}$ operating at $450 \mathrm{kHz}$ for a corresponding symmetric mode group velocity of $6000 \mathrm{~m} / \mathrm{s}$. The experimental tests provided an estimate of the relationship between the TOF variation and the micro deformations measured with a strain as follows:

(i) For $\varepsilon_{B}<1000 \mu \varepsilon$, there is a linear relationship between the TOF variation $(\Delta t)$ and the surface strain $\left(\varepsilon_{B}\right)$.

(ii) For $1000 \mu \varepsilon<\varepsilon_{B}<1400 \mu \varepsilon$, the relationship between the TOF variation and the surface strain is well approximated by a quadratic fitting. 


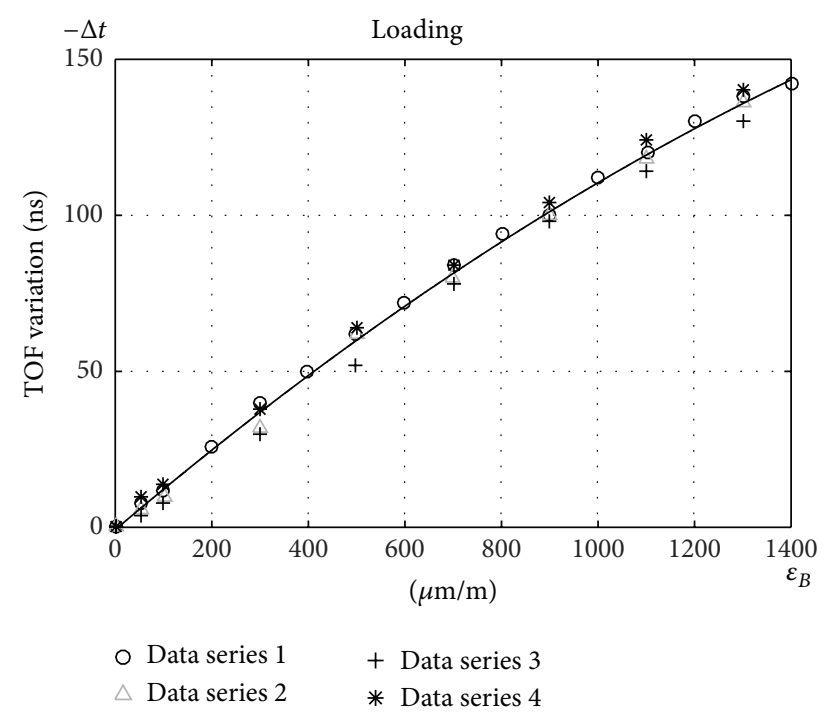

(a)

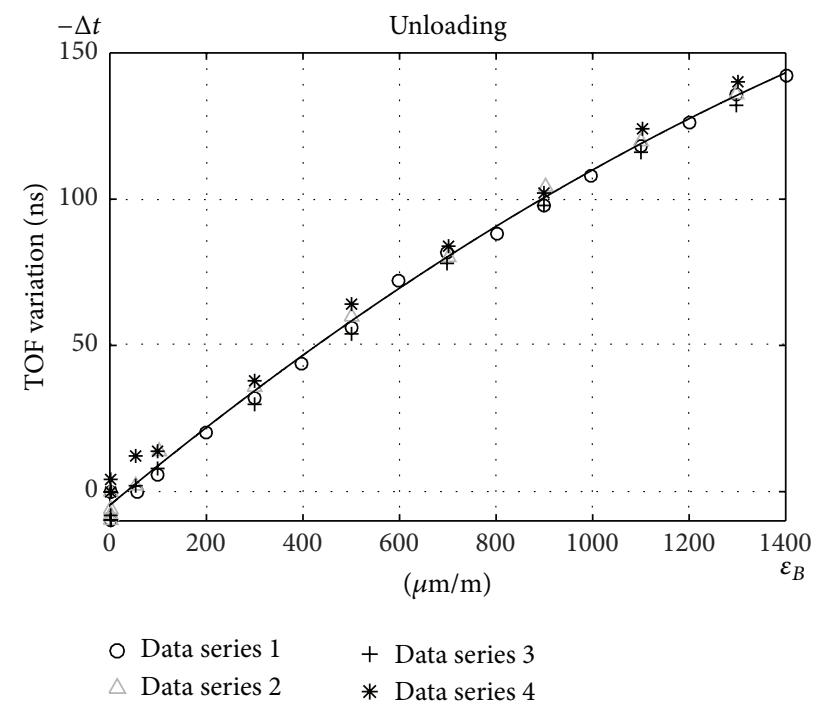

(b)

FIGURE 17: (a) Four series of data acquired in static conditions. The measurements are relative to the loading phase of pure bending stress. The continuous line shows the quadratic fitting. (b) Four series of data acquired in static conditions. The measurements are relative to the loading phase of pure bending stress. The continuous line shows the quadratic fitting.

From these measurements, we were able to estimate that the bending load standard deviation for the ultrasonic TOF method was five times greater than that of the strain gage. The TOF variation $(\Delta t)$ recorded was all negative, as observed also in another published work. We measured an increase in a single carbon fiber, using Young's dynamic modulus with an applied tensile load and also by means of ultrasonic methods. This nonlinear stress-strain response was also observed for CFRP laminates that had unidirectional and multidirectional layers.

The advantage of the piezopolymer IDTs, as compared to the strain gage, appeared when we investigated large stressed areas of the composite laminate. Moreover, we developed and tested a simple and reliable method for the acoustical coupling of piezopolymer IDTs on the sample surface based on biadhesive tape that facilitates the transducers installation and removal on the item under test.

\section{Conflict of Interests}

The authors declare that there is no conflict of interests regarding the publication of this paper.

\section{Acknowledgments}

The authors wish to acknowledge the contributions of Dr. Edgardo Rosi for providing the CFRP samples and Dr. Marina Mazzoni for the group velocity simulations.

\section{References}

[1] M. Duquennoy, M. Ouaftouh, and M. Ourak, "Ultrasonic evaluation of stresses in orthotropic materials using Rayleigh waves," NDT \& E International, vol. 32, no. 4, pp. 189-199, 1999.

[2] N. Toyama and J. Takatsubo, "An investigation of non-linear elastic behavior of CFRP laminates and strain measurement using Lamb waves," Composites Science and Technology, vol. 64, no. 16, pp. 2509-2516, 2004.

[3] C. K. Y. Leung, Z. Yang, Y. Xu, P. Tong, and S. K. L. Lee, "Delamination detection in laminate composites with an embedded fiber optical interferometric sensor," Sensors and Actuators A: Physical, vol. 119, no. 2, pp. 336-344, 2005.

[4] Y. Fan and M. Kahrizi, "Characterization of a FBG strain gage array embedded in composite structure," Sensors and Actuators, A: Physical, vol. 121, no. 2, pp. 297-305, 2005.

[5] Z. Su, L. Ye, and Y. Lu, "Guided Lamb waves for identification of damage in composite structures: a review," Journal of Sound and Vibration, vol. 295, no. 3-5, pp. 753-780, 2006.

[6] L. Capineri, A. Bulletti, M. Calzolai, and D. Francesconi, "Lamb wave ultrasonic system for active mode damage detection in composite materials," Chemical Engineering Transactions, vol. 33, pp. 577-582, 2013.

[7] L. Capineri, A. Bulletti, M. Calzolai, P. Giannelli, and D. Francesconi, "Arrays of conformable ultrasonic Lamb wave transducers for structural health monitoring with real-time electronics," in Proceedings of the 28th European Conference on Solid-State Transducers (EUROSENSORS '14), Brescia, Italy, September 2014.

[8] M. Veidt, T. Liu, and S. Kitipornchai, "Modelling of Lamb waves in composite laminated plates excited by interdigital transducers," NDT \& E International, vol. 35, no. 7, pp. 437-447, 2002.

[9] E. L. Adler, "Matrix methods applied to acoustic waves in multilayers," IEEE Transactions on Ultrasonics, Ferroelectrics, and Frequency Control, vol. 37, no. 6, pp. 485-490, 1990.

[10] N. Guo and P. Cawley, "The interaction of Lamb waves with delaminations in composite laminates," Journal of the Acoustical Society of America, vol. 94, no. 4, pp. 2240-2246, 1993.

[11] F. Bellan, A. Bulletti, L. Capineri et al., "A new design and manufacturing process for embedded Lamb waves interdigital transducers based on piezopolymer film," Sensors and Actuators A: Physical, vol. 123-124, pp. 379-387, 2005. 
[12] A. Bulletti, L. Capineri, L. Masotti, O. Occhiolini, and E. Rosi, "Strain measurements on carbon-epoxy composites by Lamb waves piezopolymer interdigital transducers," in Proceedings of the IEEE Ultrasonics Symposium, vol. 1, pp. 403-406, September 2005.

[13] S. Grondel, J. Assaad, C. Delebarre, and E. Moulin, "Health monitoring of a composite wingbox structure," Ultrasonics, vol. 42, no. 1-9, pp. 819-824, 2004.

[14] L. Capineri, L. Masotti, G. Masotti, and M. Mazzoni, "Matrixtype pyroelectric sensor, method for its fabrication and device for characterizing laser beams comprising said sensor," European Patent no. EP1380821, 2004. 

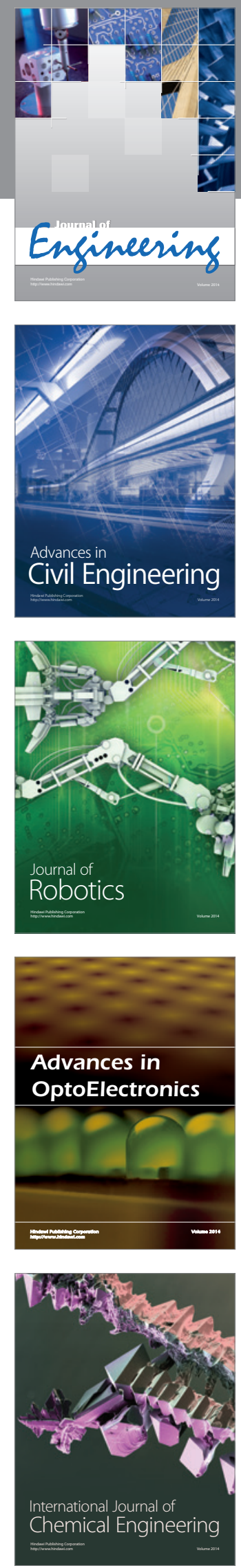

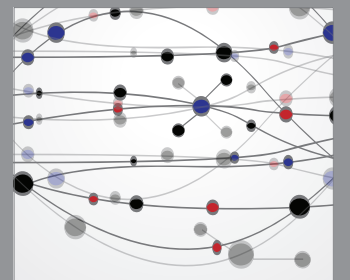

The Scientific World Journal
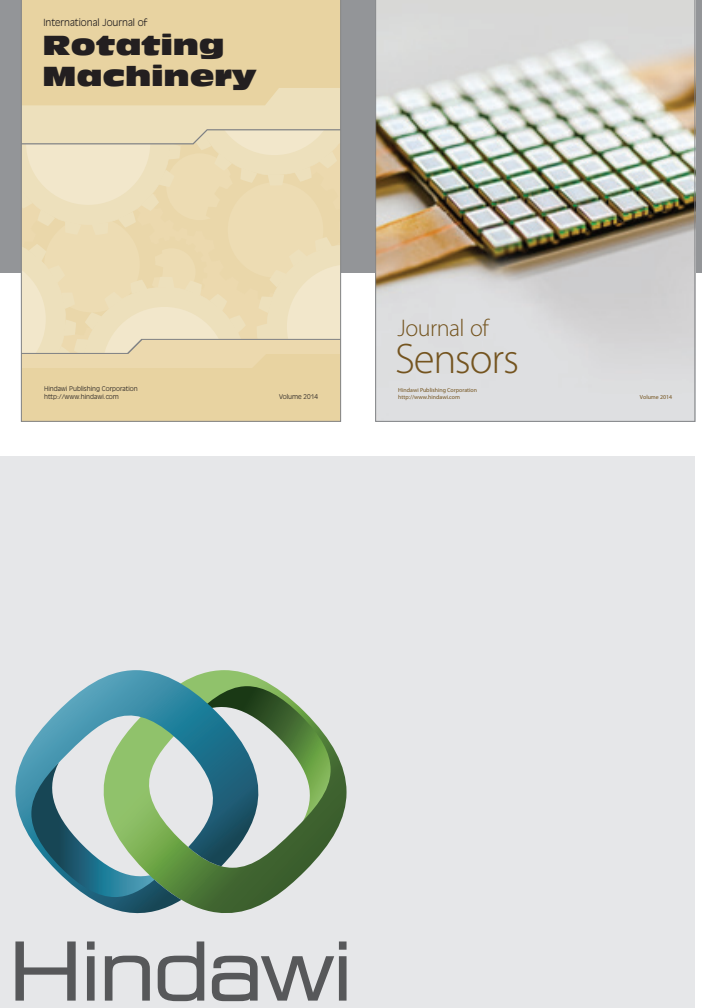

Submit your manuscripts at http://www.hindawi.com
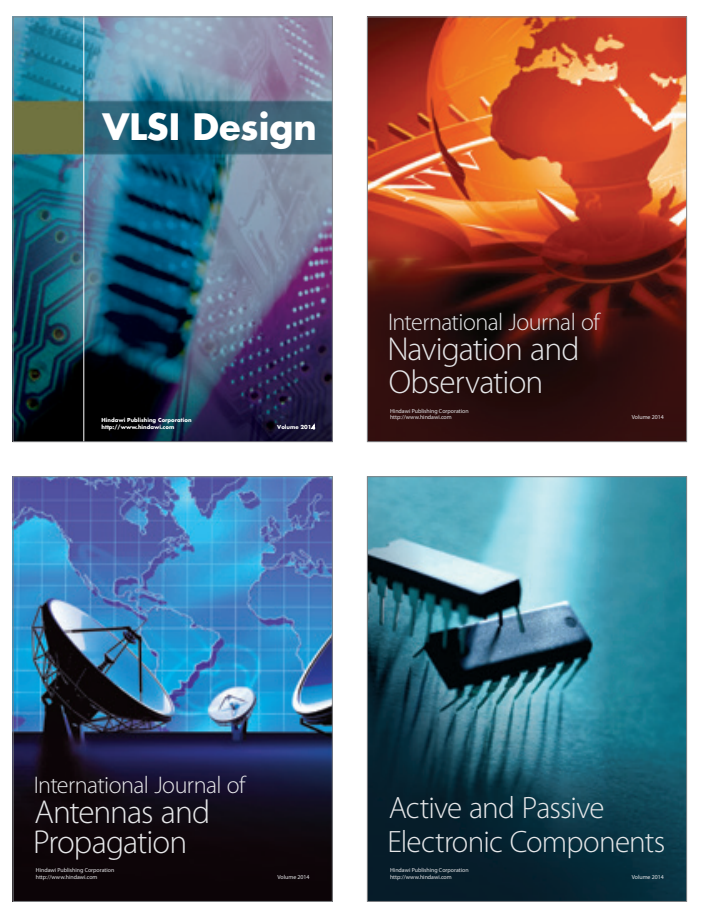
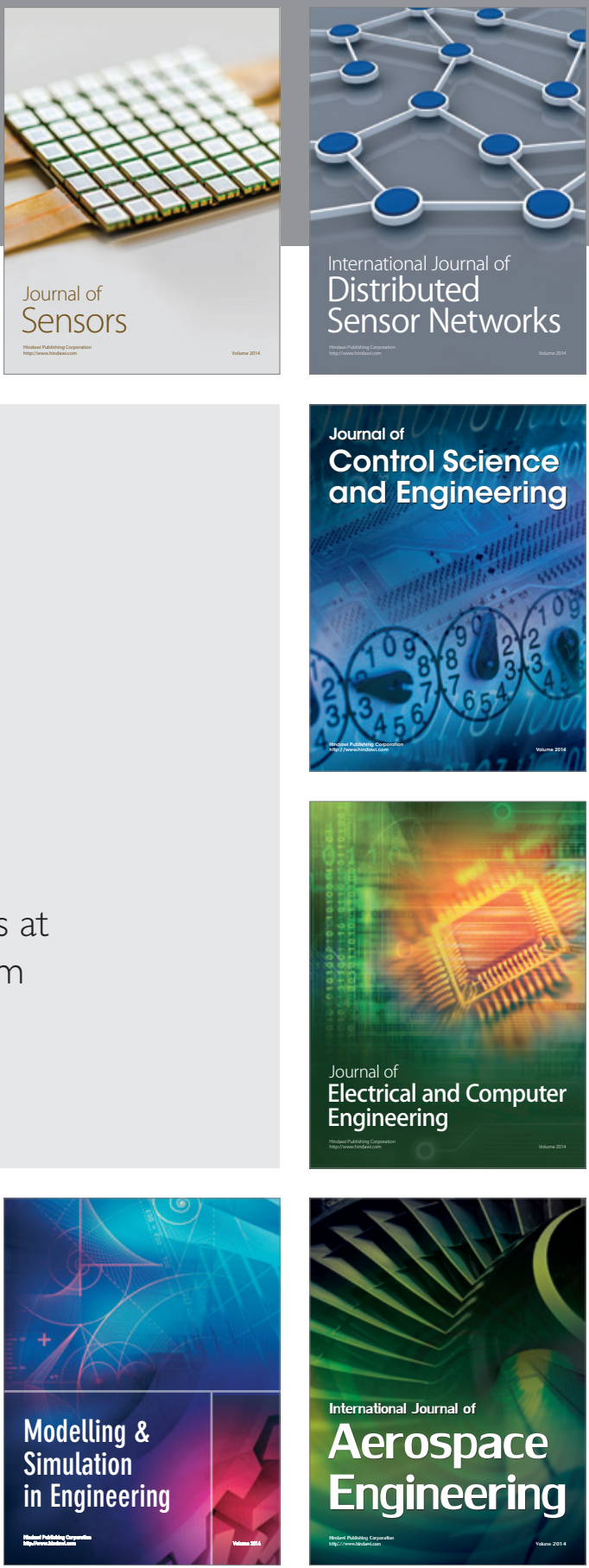

Journal of

Control Science

and Engineering
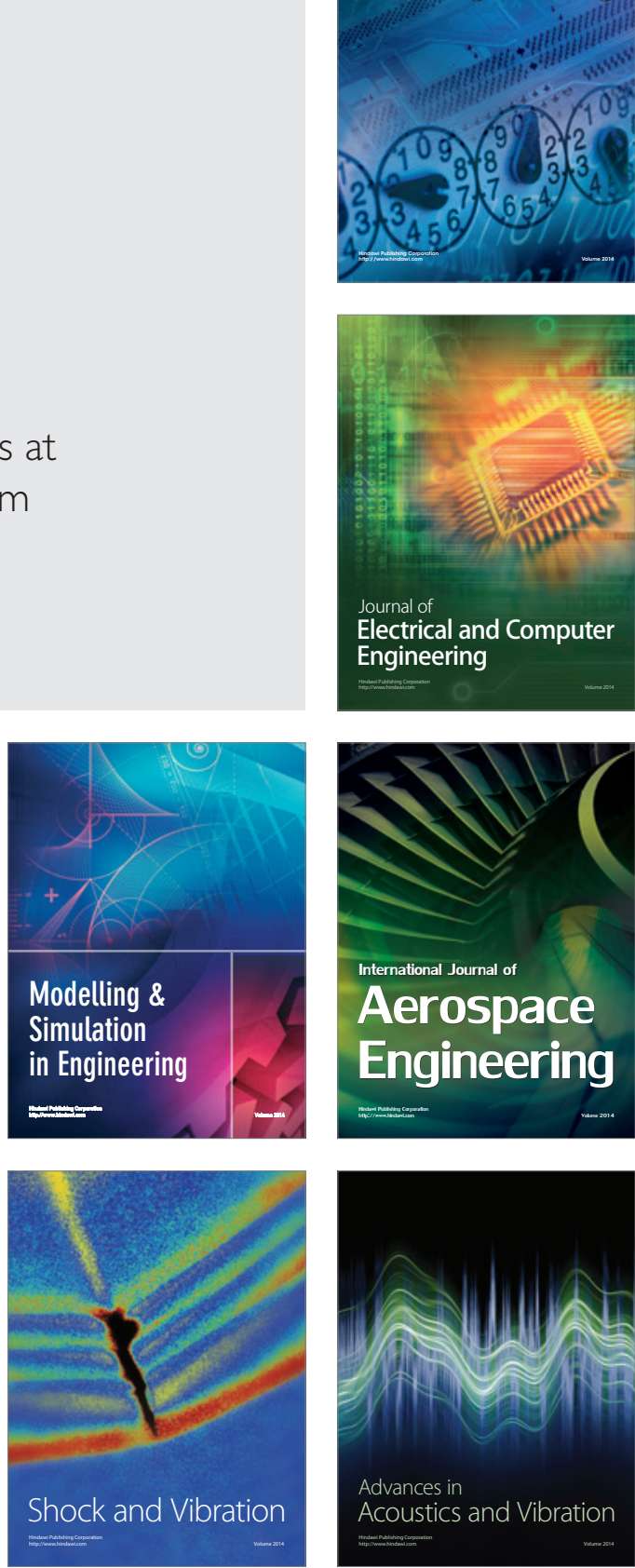